\title{
Pharmacological profiling of anti-fentanyl monoclonal antibodies in combination with naloxone in pre- and post-exposure models of fentanyl toxicity
}

Carly A. Baehr ${ }^{1}$, Mariah M. Wu ${ }^{1,2}$, Sujata G. Pandit ${ }^{3}$, Jose Arias-Umana ${ }^{3}$, David AuCoin ${ }^{3}$, and Marco Pravetoni ${ }^{1,4,5}$

${ }^{1}$ Department of Pharmacology, University of Minnesota Medical School; ${ }^{2}$ Department of Veterinary Population Medicine, University of Minnesota; ${ }^{3}$ Reno School of Medicine, University of Nevada; ${ }^{4}$ University of Minnesota Center for Immunology; ${ }^{5}$ Department of Psychiatry and Behavioral Sciences, University of Washington School of Medicine 
Running Title: Pharmacology of anti-fentanyl mAb with naloxone in rats.

Correspondence should be addressed to Carly A. Baehr; Address: 312 Church St NE, Minneapolis, MN, 55455; E-mail: cbaehr@umn.edu

Manuscript pages: 20

Figures: 6

Tables: 0

Abstract: 245 Words

Introduction: 686 Words

Discussion: 980 Words

\begin{abstract}
Abbreviations:
bpm, beats per minute

brpm, breaths per minute

SUD, substance use disorder

mAb, monoclonal antibody

NLX, naloxone

OUD, opioid use disorder

$\mathrm{SaO}_{2}$, oxygen saturation

WCS, wooden chest syndrome
\end{abstract}




\section{ABSTRACT}

The incidence of fatal drug overdoses in the United States is an alarming public health threat that has been exacerbated by the COVID-19 pandemic, resulting in over 100,000 deaths between April 2020 and April 2021. A significant portion of this is attributable to widespread access to fentanyl and other synthetic opioids, alone or in combination with heroin or psychostimulants such as cocaine or methamphetamine. Monoclonal antibodies $(\mathrm{mAb})$ offer prophylactic and therapeutic interventions against opioid overdose by binding opioids in serum, reducing distribution of drug to the brain and other organs. Here, we investigated the efficacy of a lead anti-fentanyl mAb, clone HY6-F9, in reversal and prevention of fentanyl-induced toxicity compared to the opioid receptor antagonist naloxone (NLX) in rats. In post-exposure models, rats were challenged with fentanyl, followed by HY6-F9, NLX, or both. HY6-F9 reversed fentanyl-induced antinociception, respiratory depression, and bradycardia, and rats retained protection against additional challenges for at least 1 week. Although intravenous NLX reversed fentanyl-induced respiratory depression more rapidly than mAb alone, kinetics of reversal by intravenous mAb were similar to subcutaneous NLX. Co-administration of mAb and NLX provided greater protection than individual treatments against high doses of fentanyl. Prophylactic administration of mAb reduced the $\mathrm{ED}_{50}$ of NLX approximately 2-fold against $2.25 \mathrm{mg} / \mathrm{kg}$ fentanyl. Finally, mAb sequestered fentanyl and its metabolite norfentanyl in serum, and reduced brain concentrations of fentanyl. These results support the translation of $\mathrm{mAb}$ as medical interventions alone or in combination with NLX to prevent and reverse fentanyl-related overdose.

\section{Significance statement}

Fentanyl-related overdoses have increased dramatically in the US and worldwide. Currently, approved pharmacotherapies for treatment of opioid use disorder and reversal of overdose are not sufficient to curb the incidence of opioid-related deaths. Additionally, fentanyl and its potent analogs present a potential risk from use in deliberate poisoning or chemical attacks. This study demonstrates the use of $\mathrm{mAb}$ as a countermeasure to fentanyl-induced toxicity in pre- and postexposure scenarios, supporting their use in combination with the opioid antagonist NLX. 


\section{INTRODUCTION}

Substance use disorder (SUD) and drug-related overdoses are a public health threat affecting an estimated 53 million people worldwide and resulting in 500,000 deaths each year (UNODC, 2019). In the United States, 11 million people have reported drug misuse, and 2.5 million people have been diagnosed with an opioid use disorder (OUD) (Lipari and van Horn, 2013). Unfortunately, despite the availability of pharmacological interventions for OUD, a high likelihood of relapse remains among these patients. In 2018, opioid-related fatal overdoses in the US totaled 50,000 (Hedegaard et al., 2020), and the number of non-fatal drug overdoses was 967,615 (Liu et al., 2020). These statistics have been further exacerbated by the COVID-19 pandemic caused by SARS-CoV-2, where a steep increase in opioid-related mortality to 92,600 fatal overdoses registered by early 2020 (Alter A, 2020), and passed 100,000 by April 2021 (Ahmad FB et al., 2021). The majority of drug-related deaths involve fentanyl and its analogs either alone or in mixtures with heroin, methamphetamine, or counterfeit prescription drugs (Lipari and van Horn, 2017; Hayashi et al., 2018). While approved medications are available for the treatment of OUD and reversal of overdose, it is not clear how effective these medications are in preventing and reversing accidental or deliberate overdose from fentanyl (Pergolizzi et al., 2021). Specifically, due to the high potency of fentanyl and analogs such as carfentanil, the opioid antagonist naloxone (NLX) is not as effective against fentanyl and its analogs compared to opioids such as heroin and morphine; and the short half-life of NLX may lead to re-narcotization, requiring additional NLX doses and extending hospitalization time (Rzasa Lynn and Galinkin, 2018). Additionally, fentanyl exposure can induce respiratory arrest due to rigidity of chest muscles, known as wooden chest syndrome (WCS), which is mediated by non-opioid receptor pathways and is refractory to NLX treatment (Torralva and Janowsky, 2019). Thus, more therapeutic options are needed to counteract toxicity from fentanyl-induced overdose.

As an alternative or complementary therapeutic to opioid antagonists, anti-drug monoclonal antibodies (mAb, mAbs) selectively bind the target drug in serum and extracellular fluid, reducing the concentration of unbound (free) drug in the brain and other target organs. Monoclonal antibodies are generated from antigen-specific B cell lymphocytes via hybridoma technology, by cloning and expression of recombinant mAbs, or through techniques such as phage display. In the field of SUD, murine, rodent, chimeric, humanized mAbs, and mAb fragments (F[ab'] $\left.]_{2}, \mathrm{Fab}, \mathrm{scFv}\right)$ have been reported against nicotine, cocaine, and methamphetamine (Keyler et al., 2005; Stevens et al., 2014; Wetzel et al., 2016; Marckel et al., 2019). A first-generation chimeric anti-methamphetamine mAb has completed Phase I clinical trials in healthy subjects (Stevens et al., 2014), and a second-generation anti-methamphetamine mAb (IXT-m200) has currently completed 
Phase II clinical trials in methamphetamine users (NCT 04715230). For treatment of OUD, mAbs have shown promising preclinical efficacy against heroin, oxycodone, and fentanyl in mice and rats (Kashanian et al., 2015; Kvello et al., 2019; Smith et al., 2019; Baehr et al., 2020; Ban et al., 2021), supporting further investigation of mAb as a therapeutic against overdose involving fentanyl. Previous studies have shown that opioid-specific mAbs prevent opioid-induced antinociception, respiratory depression, and bradycardia in fentanyl pre-exposure challenge models (Baehr et al., 2020), and reverse fentanyl and carfentanil-induced antinociception in post-exposure models in mice (Smith et al., 2019). The current study sought to extend previous findings by testing whether a lead anti-fentanyl ( $\alpha$-fentanyl) mAb HY6-F9, a murine $\mathrm{IgG}_{2 \mathrm{a}}$, would reverse overdose in post-exposure scenarios, by exploring the pharmacological parameters of the $\mathrm{mAb}$ alone in comparison to NLX, or in combination with NLX against doses of fentanyl from $0.1 \mathrm{mg} / \mathrm{kg}$ up to 2.25 $\mathrm{mg} / \mathrm{kg}$. The $\alpha$-fentanyl mAb provided longer protection than NLX alone, and co-administration of the mAb and NLX provided greater and longer-lasting protection against high doses of fentanyl. Treatment with mAb pre-exposure reduced the dose of NLX required to reverse fentanyl overdose measured by both oxygen saturation and antinociception. Given the longer half-life of mAb (days) compared to NLX (90 min), it is feasible to envision the use of mAb alone or in combination with NLX to reverse overdose, prevent re-narcotization, and reduce convalescence after overdose. 


\section{MATERIALS AND METHODS}

Drugs. Fentanyl citrate was obtained from the University of Minnesota Pharmacy, and naloxone hydrochloride was obtained through Sigma Aldrich (St. Louis, MO). Drug doses are expressed as concentration of free base.

Animal subjects. All animal studies were approved by the University of Minnesota Animal Care and Use Committee and conducted in AAALAC-approved facilities. Male Sprague Dawley rats (Envigo, Indianapolis, IN) were 8-10 weeks old on arrival, housed under standard conditions with a 14/10 light/dark cycle, and provided with food and water ad libitum.

Monoclonal antibody. The $\alpha$-fentanyl mAb HY6-F9 is previously described (Baehr et al., 2020). HY6-F9 is a murine $\mathrm{IgG}_{2 \mathrm{a}}$ isolated from hybridoma generated from mice immunized with a fentanyl conjugate vaccine. Antibody was harvested from hybridoma supernatant, purified with Protein A chromatography, and stored in phosphate buffered saline, $\mathrm{pH} 7.4$, at a concentration of $10 \mathrm{mg} / \mathrm{mL}$ at $4^{\circ} \mathrm{C}$.

Fentanyl challenge and reversal. Fentanyl, $0.05-0.5 \mathrm{mg} / \mathrm{mL}$, was administered subcutaneously (s.c.) at doses and time points indicated in figure legends. Naloxone, $0.1 \mathrm{mg} / \mathrm{mL}$ in saline, was administered s.c. or intravenously (i.v.) as indicated. HY6-F9, $10 \mathrm{mg} / \mathrm{mL}$, was administered i.v. in the lateral tail vein over $\sim 10 \mathrm{sec}$ with a $4.0 \mathrm{~mL} / \mathrm{kg}$ injection volume. For combination NLX + HY6-F9, NLX $0.1 \mathrm{mg} / \mathrm{mL}$ and HY6-F9 $10 \mathrm{mg} / \mathrm{mL}$ were combined at a 1:4 ratio (final concentration $0.02 \mathrm{mg} / \mathrm{mL}$ NLX and $8.0 \mathrm{mg} / \mathrm{mL}$ HY6-F9), and the admixture was administered i.v. with a $5.0 \mathrm{~mL} / \mathrm{kg}$ injection volume.

Antinociception, respiratory depression and bradycardia. Fentanyl-induced behavioral and physiological effects were measured by the hot plate test of antinociception and by pulse oximetry as previously described (Baehr et al., 2020; Robinson et al., 2020). Briefly, fentanyl-induced antinociception was evaluated by latency to respond on a hot plate set to $54^{\circ} \mathrm{C}$ (Columbus Instruments, Columbus, $\mathrm{OH}$ ). Rats were removed after displaying a lift or flick of the hind paw, or after a maximum cutoff of $30 \mathrm{~s}$ in order to avoid thermal tissue damage. Respiratory depression and bradycardia were measured with a MouseOx Plus pulse oximeter (Starr Life Sciences, Oakmont, PA) to measure oxygen saturation $\left(\mathrm{SaO}_{2}\right)$, heart rate (bmp, beats per minute), and breath rate (brpm, breaths per minute).

Fentanyl concentration in tissue. Following the completion of behavioral studies, rats were euthanized by $\mathrm{CO}_{2}$ inhalation, and trunk blood and brain were collected for analysis of fentanyl concentration. Tissue extraction was performed as previously described (Robinson et al., 2020) and fentanyl and norfentanyl concentrations were analyzed using an Agilent G6470A triple quadrupole LCMS/MS system consisting of an Infinity II 1290 G7116B Multicolumn 
Thermostat, G7120A High Speed Quad Pumps, and a G7267B Multisampler. Data acquisition and peak integration were analyzed using Mass Hunter software (Tokyo, Japan).

Statistical analysis. Statistical analyses were performed using Prism v9 (GraphPad, La Jolla, CA). Mean latency to respond, oxygen saturation, breath rate, and heart rate were analyzed by two-way ANOVA or mixed-effects analysis followed by post-hoc analyses using Sidak's or Dunnett's multiple comparisons tests. Naloxone $\mathrm{ED}_{50}$ values for latency to respond and oxygen saturation were calculated with non-linear regression, and compared between groups using extra sum-of-squares F test. 


\section{RESULTS}

\section{Reversal of fentanyl-induced antinociception, respiratory depression, and bradycardia by NLX and $\alpha$-fentanyl}

mAb HY6-F9. To determine whether $\alpha$-fentanyl mAb HY6-F9 would reverse the effects of fentanyl in vivo after fentanyl exposure, rats were challenged with $0.1 \mathrm{mg} / \mathrm{kg}$ fentanyl s.c. and monitored for fentanyl-induced antinociception, respiratory depression and bradycardia (Fig 1). Fifteen minutes after fentanyl administration, rats showed high fentanylinduced antinociception, reduced oxygen saturation, and reduced heart rate. Rats were then treated with either saline or 40 $\mathrm{mg} / \mathrm{kg}$ HY6-F9 i.v., or with $0.1 \mathrm{mg} / \mathrm{kg}$ NLX s.c. as a positive control, and monitored at 15 -minute intervals to evaluate recovery from fentanyl challenge (Fig 1). Rats treated with either HY6-F9 or NLX returned to baseline levels of antinociception and respiration within fifteen minutes after administration, and there were no statistically significant differences in efficacy of HY6-F9 compared to NLX.

To assess the duration of protection against additional fentanyl challenges, rats were challenged one week later with $0.2 \mathrm{mg} / \mathrm{kg}$ fentanyl s.c. (Fig. 2, A-D). The HY6-F9-treated rats retained protection against fentanyl-induced antinociception, respiratory depression, and bradycardia compared to rats that were previously treated with saline or NLX, and rats treated with HY6-F9 showed reduced brain distribution and high serum retention of fentanyl (Fig 2, E-F).

To evaluate the efficacy of HY6-F9 against higher doses of fentanyl, and in combination with NLX, a separate cohort of rats was challenged with a $0.5 \mathrm{mg} / \mathrm{kg}$ fentanyl s.c., and antinociception, heart rate, and respiration were measured at 15 minute intervals after fentanyl administration (Fig 3). Fifteen minutes after fentanyl administration, rats were rescued with saline control, $40 \mathrm{mg} / \mathrm{kg}$ HY6-F9 i.v., $0.1 \mathrm{mg} / \mathrm{kg}$ NLX s.c., or a combination of $40 \mathrm{mg} / \mathrm{kg}$ HY6-F9 and $0.1 \mathrm{mg} / \mathrm{kg}$ NLX i.v. At this higher dose of fentanyl, rats treated with HY6-F9, NLX, or the combination showed increased oxygen saturation and reduced fentanyl-induced antinociception compared to saline control within 30-45 min of administration.

One week later, rats were challenged with $0.6 \mathrm{mg} / \mathrm{kg}$ fentanyl s.c. (Suppl. Fig 1). After the first measurement at 15 min, rats that received either saline or HY6-F9 the previous week received saline i.v., while rats that previously received NLX or NLX + HY6-F9 received an injection of $0.1 \mathrm{mg} / \mathrm{kg}$ NLX s.c. HY6-F9 alone was not effective against this dose of fentanyl, NLX alone was partially effective in reversing fentanyl-induced effects, and rats treated with the combination of NLX + HY6-F9 showed the greatest protection against the effects of fentanyl. Four hours after the second challenge, rats were given a final challenge of $0.4 \mathrm{mg} / \mathrm{kg}$ fentanyl (Suppl. Fig 2). Fentanyl distribution to brain and serum were measured 
one hour after the final fentanyl challenge, and rats treated with HY6-F9 showed reduced brain distribution of fentanyl compared to control (Suppl. Fig 2D-F).

Kinetics of respiratory depression reversal by HY6-F9 compared to i.v. or s.c. NLX. To determine the rate of onset of HY6-F9 compared to NLX, rats were given a challenge of $0.1 \mathrm{mg} / \mathrm{kg}$ fentanyl. Fifteen minutes after fentanyl administration, rats were monitored for oxygen saturation during and after rescue with saline, HY6-F9 i.v., NLX either s.c. or i.v., or an i.v. mixture of NLX + HY6-F9 (Fig 4B). While intravenous NLX and the combination NLX + HY6-F9 reversed fentanyl-induced respiratory depression within $\sim 1$ minute, i.v. HY6-F9 alone reversed fentanyl effects in $\sim 2-4$ min. By comparison, s.c. naloxone reversed fentanyl effects in $\sim 2$ min. Antinociception (Fig 4A), heart rate (Fig 4C) and breath rate (Fig 4D) were measured 15 minutes after fentanyl administration and 15 minutes after rescue.

Pharmacokinetic profiling of NLX and HY6-F9 efficacy against high fentanyl doses. To evaluate the efficacy of NLX compared to the combination of NLX $+\mathrm{mAb}$ against increasing doses of fentanyl in pre-exposure scenarios, rats were pre-treated with saline or $40 \mathrm{mg} / \mathrm{kg} \mathrm{HY6-F9.} \mathrm{Fifteen} \mathrm{minutes} \mathrm{prior} \mathrm{to} \mathrm{challenge,} \mathrm{rats} \mathrm{received} \mathrm{saline} \mathrm{or} 0.1 \mathrm{mg} / \mathrm{kg} \mathrm{NLX}$, and then increasing doses of fentanyl were administered s.c. at 15 -min intervals, up to a cumulative dose of $0.5 \mathrm{mg} / \mathrm{kg}$ (Fig 5A-B). HY6-F9 alone was effective at preventing fentanyl-induced antinociception and hypoxemia at $0.1 \mathrm{mg} / \mathrm{kg}$ fentanyl and partially effective at $0.25 \mathrm{mg} / \mathrm{kg}$, but was not effective at a cumulative dose of $0.5 \mathrm{mg} / \mathrm{kg}$ fentanyl. NLX alone was effective at preventing fentanyl-induced hypoxemia at $0.1-0.5 \mathrm{mg} / \mathrm{kg}$ fentanyl, but was not effective against fentanyl-induced antinociception at $0.5 \mathrm{mg} / \mathrm{kg}$ fentanyl. By contrast, the combination of NLX + HY6-F9 prevented both fentanyl-induced antinociceptive and respiratory effects up to $0.5 \mathrm{mg} / \mathrm{kg}$ fentanyl.

Because NLX is a competitive inhibitor of opioid receptor, whereas $\alpha$-fentanyl mAb affects distribution of fentanyl, we hypothesized that prophylactic administration of mAb would shift the dose-response curve for NLX against high fentanyl doses by reducing the concentration of fentanyl competing against NLX for opioid receptor binding in the brain. Rats were pre-treated with saline or with $10-40 \mathrm{mg} / \mathrm{kg}$ HY6-F9, then challenged with $2.25 \mathrm{mg} / \mathrm{kg}$ fentanyl s.c., which is near the reported $\mathrm{LD}_{50}$ for this drug (Vardanyan and Hruby, 2014). Starting at 10 min after fentanyl administration, rats were given increasing doses of NLX s.c., and antinociception and oxygen saturation were measured at 4-min intervals to evaluate reversal of fentanyl toxicity. In saline-treated rats, approximately $0.3 \mathrm{mg} / \mathrm{kg}$ NLX was required to reverse fentanyl-induced hypoxemia, defined by $\mathrm{SaO}_{2}>90 \%$ (Fig 5D); and $1.0 \mathrm{mg} / \mathrm{kg}$ NLX partially reduced fentanyl-

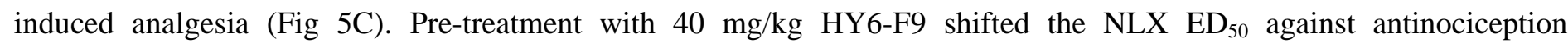
approximately 3 -fold $\left(\mathrm{ED}_{50}=1.362 \mathrm{mg} / \mathrm{kg}\right.$ for NLX vs 0.4608 for $\left.\mathrm{NLX}+40 \mathrm{mg} / \mathrm{kg} \mathrm{HY} 6-\mathrm{F} 9, p=0.0116\right)$, and shifted the 
NLX ED ${ }_{50}$ for oxygen saturation 2-fold $\left(\mathrm{ED}_{50}=0.07398 \mathrm{mg} / \mathrm{kg}\right.$ for NLX vs 0.02906 for NLX $+40 \mathrm{mg} / \mathrm{kg} \mathrm{HY} 6-\mathrm{F9}$, $p=0.0129$ ). Notably, a NLX dose sufficient to reverse hypoxemia was not sufficient for rats to achieve consciousness (data not shown); rats treated with only NLX regained consciousness an average of 27.5 min after fentanyl administration (i.e. 1-2 min after a dose of $1.0 \mathrm{mg} / \mathrm{kg} \mathrm{NLX}$ ), whereas rats treated with $40 \mathrm{mg} / \mathrm{kg} \mathrm{HY} 6-\mathrm{F} 9$ regained consciousness an average of $24 \mathrm{~min}$ after fentanyl administration (i.e. 2 min after a dose of $0.3 \mathrm{mg} / \mathrm{kg} \mathrm{NLX}$ ). A dose of $10 \mathrm{mg} / \mathrm{kg} \mathrm{HY6-F9}$ shifted the NLX ED $\mathrm{E}_{50}$ for oxygen saturation, but did not reduce fentanyl-induced antinociception nor time or dose required for consciousness compared to NLX alone, suggesting that the higher $\mathrm{mAb}$ dose of $40 \mathrm{mg} / \mathrm{kg}$ is necessary to increase NLX efficacy against this dose of fentanyl.

Following the $0.5 \mathrm{mg} / \mathrm{kg}$ cumulative fentanyl challenge (Fig 5A-B), serum and brain of fentanyl-treated rats were collected for analysis of fentanyl and norfentanyl concentrations (Fig 6). Rats treated with either HY6-F9 or NLX + HY6F9 showed significantly increased serum concentrations of fentanyl, and reduced concentrations of fentanyl in brain (Fig 6A-B). Similarly, HY6-F9-treated rats showed increased serum concentrations and reduced brain concentrations of the fentanyl metabolite norfentanyl (Fig 6C-D). Surprisingly, rats treated with NLX alone showed a significant increase in brain norfentanyl compared to rats treated with saline (Fig 6D), but NLX did not affect serum norfentanyl compared to $\operatorname{control}(p=0.58)$. 


\section{DISCUSSION}

As the incidence of opioid-related fatal overdoses continues to rise in the US, new therapeutic and prophylactic strategies are essential for public health and safety. Currently, opioid antagonists such as NLX remain the main defense against opioid-related overdoses; however, particularly in the case of fentanyl, it is unclear how effective these antagonists are in preventing and reversing fentanyl-related overdose (Hill et al., 2020; Pergolizzi et al., 2021). This highlights the importance of identifying new therapeutic options to counteract fentanyl overdose. Monoclonal antibodies offer a promising tool for the treatment of OUD and prevention of opioid-related toxicity and overdose; however, previous studies evaluating efficacy of $\alpha$-fentanyl mAb have been limited in the mAb and fentanyl doses used, and have not directly demonstrated the efficacy of mAb over time against repeated fentanyl exposure. In pre-clinical studies, passive immunization with doses of $30-120 \mathrm{mg} / \mathrm{kg}$ of $\alpha$-opioid mAb were shown to counteract the pharmacological effects of fentanyl (Smith et al., 2019; Baehr et al., 2020; Ban et al., 2021), and reversal of fentanyl effects post-exposure has only been demonstrated 30-60 minutes after administration (Smith et al., 2019). The present study expands upon previous results by demonstrating that not only does HY6-F9 rapidly reverse the effects of $0.1 \mathrm{mg} / \mathrm{kg}$ fentanyl post-exposure within $5 \mathrm{~min}$, but also that prophylactic exposure to $\mathrm{mAb}$ protects against further fentanyl challenges at least 1 week after initial $\mathrm{mAb}$ treatment. Additionally, previous studies of $\alpha$-opioid mAb generally utilize opioid-induced antinociception as the primary measure of $\mathrm{mAb}$ efficacy, whereas fentanyl-induced respiratory depression is a major contributor in overdoserelated fatalities (Dolinak, 2017). Hence, this study measured fentanyl-induced hypoxemia and bradycardia in addition to antinociception, offering more clinically relevant parameters for evaluating efficacy of biologics against fentanyl.

Additionally, these results demonstrate that HY6-F9 enhances efficacy of NLX against relatively high fentanyl doses. The increasing incidence of fentanyl-related overdose fatalities indicates that NLX alone at its clinically utilized dose of 2-10 mg may be insufficient to counteract fentanyl overdose; and simulated pharmacokinetic modeling suggest that as fentanyl concentration increases, NLX is able to displace a lower proportion of fentanyl from $\mu$ opioid receptors (Moss Id et al., 2020), consistent with its competitive antagonist mechanism. By contrast, the pharmacokinetic mechanism of mAb alters distribution of fentanyl, thus decreasing concentration of fentanyl available for receptor interaction. Hence, mAb enhances efficacy of NLX by reducing brain fentanyl levels below a concentration at which NLX is able to outcompete fentanyl for receptors. Here, HY6-F9 increased NLX efficacy in both a cumulative fentanyl challenge up to 0.5 $\mathrm{mg} / \mathrm{kg}$, and in a high dose fentanyl challenge of $2.25 \mathrm{mg} / \mathrm{kg}$ combined with NLX dose-response (Fig 5). The stoichiometric mechanism of $\alpha$-fentanyl mAb sequestration suggests that efficacy of mAb is highly dependent on the ratio 
of available fentanyl-binding mAb to circulating drug; here, a dose of $40 \mathrm{mg} / \mathrm{kg}$ HY6-F9 reduced brain fentanyl concentration by $>90 \%$ during a challenge of $0.2 \mathrm{mg} / \mathrm{kg}$ (Fig 2), and by $70 \%$ during the cumulative challenge of 0.5 $\mathrm{mg} / \mathrm{kg}$ (Fig 6). Although HY6-F9 alone did not prevent fentanyl-induced effects at a dose of 0.2-0.5 mg/kg fentanyl, the significant reduction in brain fentanyl at this dose indicate potential utility of $\mathrm{mAb}$ in reducing overall exposure of opioid receptors to fentanyl and/or increasing efficacy of opioid antagonists at high opioid doses, supported by the observation that the combination of $40 \mathrm{mg} / \mathrm{kg}$ HY6-F9 and $0.1 \mathrm{mg} / \mathrm{kg}$ NLX prevented antinociception above $0.5 \mathrm{mg} / \mathrm{kg}$ fentanyl.

Fentanyl-related fatal overdoses have also been associated with WCS, in which the chest wall and upper airways become rigid in response to fentanyl exposure, both impairing respiration and compromising the ability of first responders to conduct CPR in overdose patients. While the mechanisms underlying WCS are not entirely understood, it appears that WCS is primarily mediated by alpha-adrenergic and cholinergic signaling rather than opioid receptor signaling (Torralva and Janowsky, 2019). In contrast to morphine and other opioids, fentanyl has demonstrated interaction with non-opioid receptors including adrenoceptors (Torralva et al., 2020). Hence, NLX or other opioid receptor-specific pharmacotherapy is not effective in reversing WCS (Miner et al., 2021). Instead, reducing the concentration of free (unbound) fentanyl through vaccine-induced polyclonal antibodies or $\alpha$-opioid mAbs may be particularly beneficial in limiting the occurrence of fentanyl-induced WCS by reducing overall signaling to all fentanyl-responsive receptors in both central and peripheral nervous system.

Several known or potential limitations of mAb-based therapeutics targeting small molecules remain to be explored. First, the high doses of $\mathrm{mAb}$ and apparent requirement of i.v. administration for efficacy post-fentanyl exposure could render clinical application difficult. In support for accelerating translation, future studies will need to explore mAb formulations suitable for s.c. or i.m. delivery, which may broaden feasibility of administering mAb in clinical settings. Additionally, while high specificity of mAb for fentanyl may be beneficial by allowing concurrent administration of nonfentanyl opioids, it may limit utility of mAb as a reversal agent in cases of multi-opioid exposure or exposure to fentanyl analogs. However, as demonstrated by a previously described anti-fentanyl mAb 6A4 that showed efficacy against both fentanyl and carfentanil in mice (Smith et al., 2019), it is possible to envision development of mAbs that cross-react with a variety of compounds within the fentanyl-like chemical family. Potentially, clinical efficacy against multiple compounds could be achieved by co-administration of multiple mAbs targeting a series of structurally distinct opioids. Finally, although naloxone for overdose reversal exhibits a short half-life, extended release formulations exist of the antagonist naltrexone which would exhibit similarly long duration of efficacy to mAb; however, such medications may complicate 
management of withdrawal symptoms and other side effects. While it has not been tested whether anti-fentanyl mAb would precipitate withdrawal in opioid-dependent individuals, previous work with anti-nicotine mAb showed no mAbinduced withdrawal effects (Roiko et al., 2009). These data support further evaluation of mAbs as a therapeutic and prophylactic strategy to limit the occurrence of OUD and opioid related overdose and support their use alongside FDAapproved medications such as NLX. 
Acknowledgements: The authors thank Jennifer Vigliaturo and Diego Luengas for technical support.

\section{Authorship contributions:}

Participated in research design: Baehr, Pravetoni

Conducted experiments and performed data analysis: Baehr, $\mathrm{Wu}$

Contributed new reagents or analytical tools: Pandit, Arias-Umana

Contributed to writing of the manuscript: Baehr, Wu, AuCoin, Pravetoni 


\section{References}

Ahmad FB, Rossen LM, and Sutton P (2021) Provisional drug overdose death counts.

Alter A YC (2020) COVID-19 Impact on US National Overdose Crisis. Overdose Detection Mapping Application

Program.

Baehr C, Kelcher AH, Khaimraj A, Reed DE, Pandit SG, AuCoin D, Averick S, and Pravetoni M (2020) Monoclonal Antibodies Counteract Opioid-Induced Behavioral and Toxic Effects in Mice and Rats. Journal of Pharmacology and Experimental Therapeutics 375:469-477, American Society for Pharmacology and Experimental Therapy.

Ban B, Barrientos RC, Oertel T, Komla E, Whalen C, Sopko M, You Y, Banerjee P, Sulima A, Jacobson AE, Rice KC, Matyas GR, and Yusibov V (2021) Novel chimeric monoclonal antibodies that block fentanyl effects and alter fentanyl biodistribution in mice. $m A b s \mathbf{1 3}$, Taylor \& Francis.

Dolinak D (2017) Opioid Toxicity, SAGE Publications Inc.

Hayashi K, Milloy M-J, Lysyshyn M, DeBeck K, Nosova E, Wood E, and Kerr T (2018) Substance use patterns associated with recent exposure to fentanyl among people who inject drugs in Vancouver, Canada: A cross-sectional urine toxicology screening study. Drug and alcohol dependence 183:1, NIH Public Access.

Hedegaard H, Miniño AM, and Warner M (2020) Drug Overdose Deaths in the United States, 1999-2018 Key findings Data from the National Vital Statistics System, Mortality.

Hill R, Santhakumar R, Dewey W, Kelly E, and Henderson G (2020) Fentanyl depression of respiration: Comparison with heroin and morphine. British Journal of Pharmacology 177:254-266, John Wiley and Sons Inc.

Kashanian S, Shams A, Ghahremani H, and Paknejad M (2015) Preparation and Characterization of a Monoclonal Antibody Against Morphine. Monoclonal Antibodies in Immunodiagnosis and Immunotherapy 34:270-274, Mary Ann Liebert Inc.

Keyler DE, Roiko SA, Benlhabib E, LeSage MG, st. Peter J v, Stewart S, Fuller S, Le CT, and Pentel PR (2005) Monoclonal Nicotine-specific Antibodies Reduce Nicotine Distribution to Brain in Rats: Dose- and Affinityresponse Relationships. Drug Metabolism and Disposition 33:1056.

Kvello AMS, Andersen JM, Øiestad EL, Steinsland S, Aase A, Mørland J, and Bogen IL (2019) A monoclonal antibody against 6-acetylmorphine protects female mice offspring from adverse behavioral effects induced by prenatal heroin exposure. Journal of Pharmacology and Experimental Therapeutics 368:106-115, American Society for Pharmacology and Experimental Therapy. 
Lipari RN, and van Horn SL (2013) Trends in Substance Use Disorders Among Adults Aged 18 or Older.

Lipari RN, and van Horn SL (2017) Trends in Substance Use Disorders Among Adults Aged 18 or Older.

Liu S, Scholl L, Hoots B, and Seth P (2020) Nonfatal Drug and Polydrug Overdoses Treated in Emergency Departments

— 29 States, 2018-2019. MMWR Morbidity and Mortality Weekly Report 69:1149-1155, Centers for Disease

Control MMWR Office.

Marckel JA, Wetzel HN, Amlal S, Amlal H, and Norman AB (2019) A Recombinant Humanized Anticocaine

Monoclonal Antibody Alters the Urinary Clearance of Cocaine and Its Metabolites in Rats. Drug metabolism and disposition: the biological fate of chemicals 47:184-188, The American Society for Pharmacology and Experimental Therapeutics.

Miner NB, Schutzer WE, Zarnegarnia Y, Janowsky A, and Torralva R (2021) Fentanyl causes naloxone-resistant vocal cord closure: A platform for testing opioid overdose treatments. Drug and Alcohol Dependence 227:108974, Drug Alcohol Depend.

Moss Id RB, Mccabe Pryor M, Baillie R, Kudrycki K, Friedrich C, Reed M, and Carlo DJ (2020) Higher naloxone dosing in a quantitative systems pharmacology model that predicts naloxone-fentanyl competition at the opioid mu receptor level. , doi: 10.1371/journal.pone.0234683.

Pergolizzi J v., Dahan A, Ann LeQuang J, and Raffa RB (2021) Overdoses due to fentanyl and its analogues (F/FAs) push naloxone to the limit. Journal of Clinical Pharmacy and Therapeutics 46:1501-1504, J Clin Pharm Ther.

Robinson C, Gradinati V, Hamid F, Baehr C, Crouse B, Averick S, Kovaliov M, Harris D, Runyon S, Baruffaldi F,

LeSage M, Comer S, and Pravetoni M (2020) Therapeutic and Prophylactic Vaccines to Counteract Fentanyl Use Disorders and Toxicity. Journal of Medicinal Chemistry 63:14647-14667, American Chemical Society.

Roiko SA, Harris AC, LeSage MG, Keyler DE, and Pentel PR (2009) Passive immunization with a nicotine-specific monoclonal antibody decreases brain nicotine levels but does not precipitate withdrawal in nicotine-dependent rats. Pharmacology, biochemistry, and behavior 93:105, NIH Public Access.

Rzasa Lynn R, and Galinkin JL (2018) Naloxone dosage for opioid reversal: current evidence and clinical implications, SAGE Publications Ltd.

Smith LC, Bremer PT, Hwang CS, Zhou B, Ellis B, Hixon MS, and Janda KD (2019) Monoclonal Antibodies for Combating Synthetic Opioid Intoxication. Journal of the American Chemical Society, doi: 10.1021/jacs.9b04872. 
Stevens MW, Henry RL, Owens SM, Schutz R, and Gentry WB (2014) First human study of a chimeric antimethamphetamine monoclonal antibody in healthy volunteers. mAbs 6:1649-1656, Taylor \& Francis.

Torralva R, Eshleman AJ, Swanson TL, Schmachtenberg JL, Schutzer WE, Bloom SH, Wolfrum KM, Reed JF, and Janowsky A (2020) Fentanyl but not Morphine Interacts with Nonopioid Recombinant Human Neurotransmitter Receptors and Transporters. Journal of Pharmacology and Experimental Therapeutics 374:376-391.

Torralva R, and Janowsky A (2019) Noradrenergic Mechanisms in Fentanyl-Mediated Rapid Death Explain Failure of Naloxone in the Opioid Crisis. Journal of Pharmacology and Experimental Therapeutics 371:453-475.

UNODC (2019) World Drug Report 2019 Methodology Report, UN.

Vardanyan RS, and Hruby VJ (2014) Fentanyl-related compounds and derivatives: current status and future prospects for pharmaceutical applications. Future Medicinal Chemistry 6:385-412.

Wetzel HN, Tsibulsky VL, and Norman AB (2016) The effects of a repeated dose of a recombinant humanized anticocaine monoclonal antibody on cocaine self-administration in rats. Drug and alcohol dependence 168:287-292. 


\section{Footnotes}

Financial support: This work was supported by the National Institute on Drug Abuse, National Institute of Allergy and Infectious Diseases, and the Office of the Director of the National Institutes of Health under CounterACT award number [Grant U01DA051658] (to M.P.); and by the National Institutes of Health under [Grant T32DA007097] (to M.M.W.). The content is solely the responsibility of the authors and does not necessarily represent the official views of the National Institutes of Health.

Disclosures: M.P. and C.B. are inventors of a patent application "Anti-opioid compounds and methods of making and using same." All other authors declare no conflicts of interest. 


\section{Figure Legends}

Figure 1. Anti-fentanyl mAb HY6-F9 reverses pharmacological effects of fentanyl. Sprague Dawley rats ( $\mathrm{n}=2-3$ per group) were challenged with $0.1 \mathrm{mg} / \mathrm{kg}$ fentanyl (s.c.) and monitored for fentanyl-induced antinociception, respiratory depression and bradycardia. (A) antinociception via latency to respond on the hot plate test; and (B) oxygen saturation, (C) bradycardia, and (D) breath rate measured by pulse oximetry. Immediately following the first measurement at $15 \mathrm{~min}$, rats were passively immunized with $40 \mathrm{mg} / \mathrm{kg}$ HY6-F9 (i.v.) or injected with saline (i.v.) or $0.1 \mathrm{mg} / \mathrm{kg}$ NLX (s.c.) as a positive control. Data are mean \pm SEM. $* * * *<<0.0001,{ }^{*} * p<0.01, * p<0.05$ relative to saline (control).

Figure 2. HY6-F9 protects against further fentanyl challenges. One week after initial fentanyl challenge, rats (Fig. 1) were challenged with $0.2 \mathrm{mg} / \mathrm{kg}$ fentanyl (s.c.), and monitored for (A) antinociception, (B) oxygen saturation, (C) heart rate, and (D) breath rate. At 30 minutes post-fentanyl, fentanyl concentration was measured in (E) serum and (F) brain. Data are mean \pm SEM. $* * * * p<0.0001, * * * p<0.001, * * p<0.01, * p<0.05$ relative to saline (control).

Figure 3. Co-administration of HY6-F9 and NLX reverses effects of high dose fentanyl. A second cohort of naïve rats ( $\mathrm{n}=3$ per group) was challenged with $0.5 \mathrm{mg} / \mathrm{kg}$ fentanyl (s.c.) and monitored for fentanyl-induced antinociception, respiratory depression and bradycardia. Immediately following the first measurement at 15 min, rats were passively immunized with NLX s.c., HY6-F9 i.v., or a combination of NLX and mAb i.v. (A) Antinociception measured as latency to respond, and (B) oxygen saturation, (C) heart rate (beats per minute, bpm), and (D) breath rate (breaths per minute, brpm) measured by pulse oximetry. Data are mean \pm SEM. $* * p<0.01, * p<0.05$ relative to saline (control).

Figure 4. NLX and HY6-F9 rapidly reverse fentanyl-induced oxygen saturation. Naïve rats were challenged with 0.1 mg/kg fentanyl (s.c.), and 15 minutes post-fentanyl exposure, rats were given saline, NLX either i.v. or s.c., HY6-F9 i.v., or NLX + HY6-F9 i.v.. (A) Antinociception was measured 15 and 30 min post-fentanyl exposure, and (B) oxygen saturation measured constantly by pulse oximetry for $10 \mathrm{~min}$ after rescue administration. (C) Inset to show detail of oxygen saturation from 15-20 min after fentanyl administration.

Figure 5. Pre-exposure NLX or HY6-F9 in cumulative fentanyl challenge and NLX dose-response. A-B, Rats pretreated with $40 \mathrm{mg} / \mathrm{kg} \mathrm{mAb}$ were treated with saline or $0.1 \mathrm{mg} / \mathrm{kg}$ NLX s.c. Fifteen minutes after NLX, rats were challenged at 15-minute intervals with increasing doses of fentanyl, to a cumulative dose of $0.5 \mathrm{mg} / \mathrm{kg}$. (A) Antinociception measured as latency to respond, and (B) oxygen saturation measured by pulse oximetry. C-D, Rats pretreated with 10 or $40 \mathrm{mg} / \mathrm{kg} \mathrm{mAb}$ were given $2.25 \mathrm{mg} / \mathrm{kg}$ fentanyl. Ten minutes after fentanyl, and at 4-minute intervals, rats were given increasing doses of NLX to reverse overdose. (C) Antinociception measured as latency to respond, and 
(D) oxygen saturation measured by pulse oximetry. Data are mean \pm SEM. ${ }^{*} * * * p<0.0001, * * * p<0.001, * * p<0.01$, $* p<0.05$ relative to saline (control).

Figure 6. HY6-F9 sequesters fentanyl and norfentanyl in serum. After the final dose of fentanyl (Fig 5A-B), blood and brain were collected for analysis of concentration of fentanyl and its metabolites in tissue. Fentanyl concentration in (A) serum and (B) brain. Norfentanyl concentration in (D) serum and (E) brain. Data are mean \pm SEM. $* * * * p<0.0001$, $* * * p<0.001, * * p<0.01$ relative to saline (control), or bars to indicate significance between groups. 


\section{Figure 1}

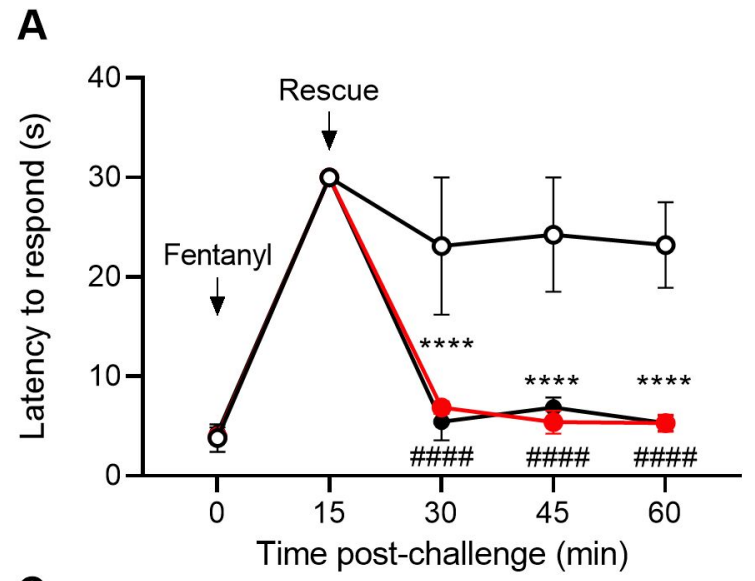

C
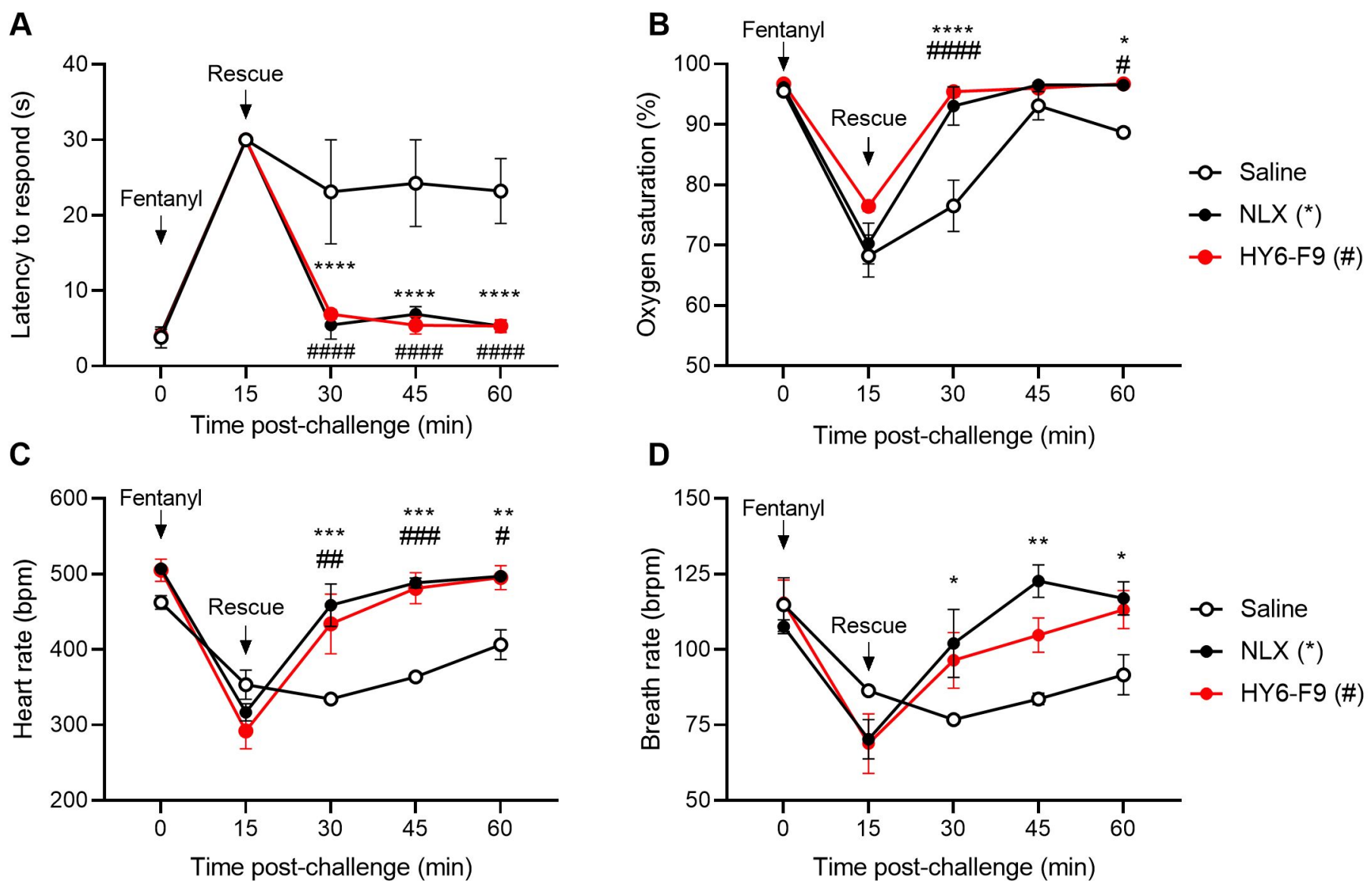


\section{Figure 2}

A

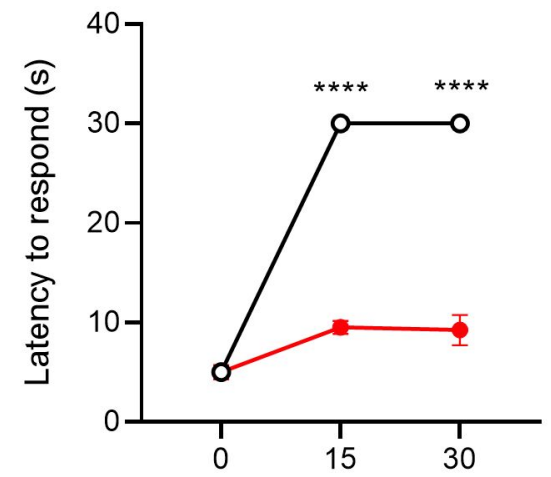

Time post-challenge (min)

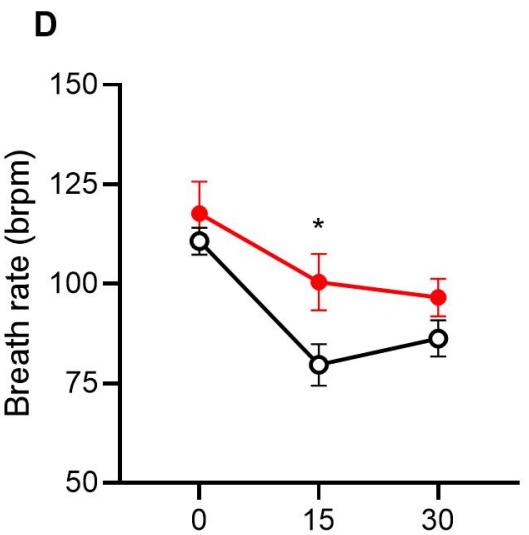

B

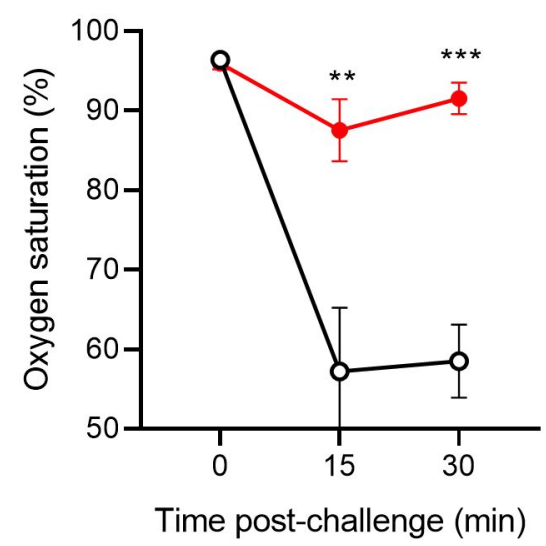

E

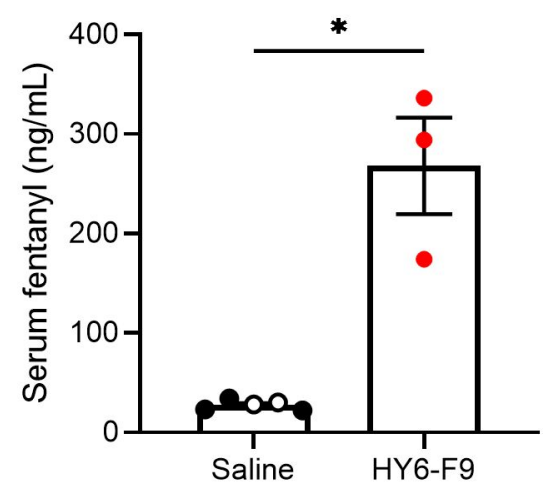

\section{C}

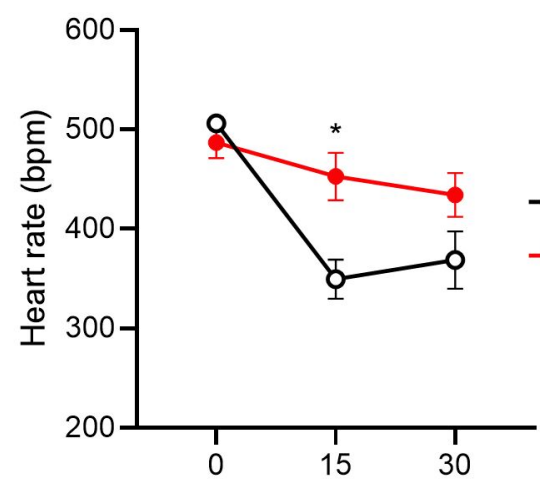

Time post-challenge ( $\mathrm{min})$

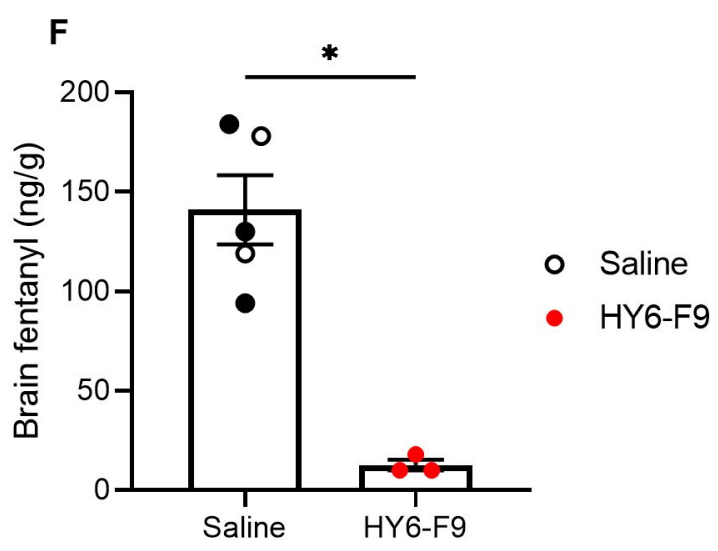

Time post-challenge ( $\mathrm{min}$ ) 


\section{Figure 3}
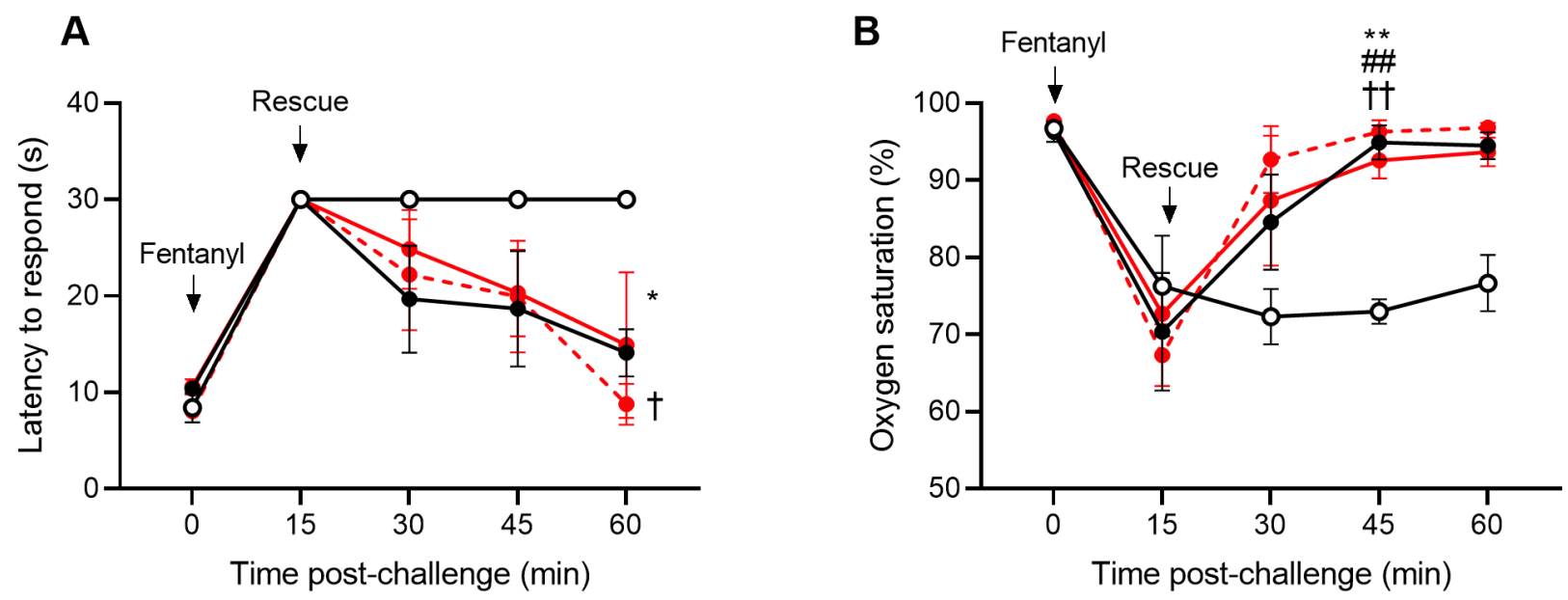

C

D
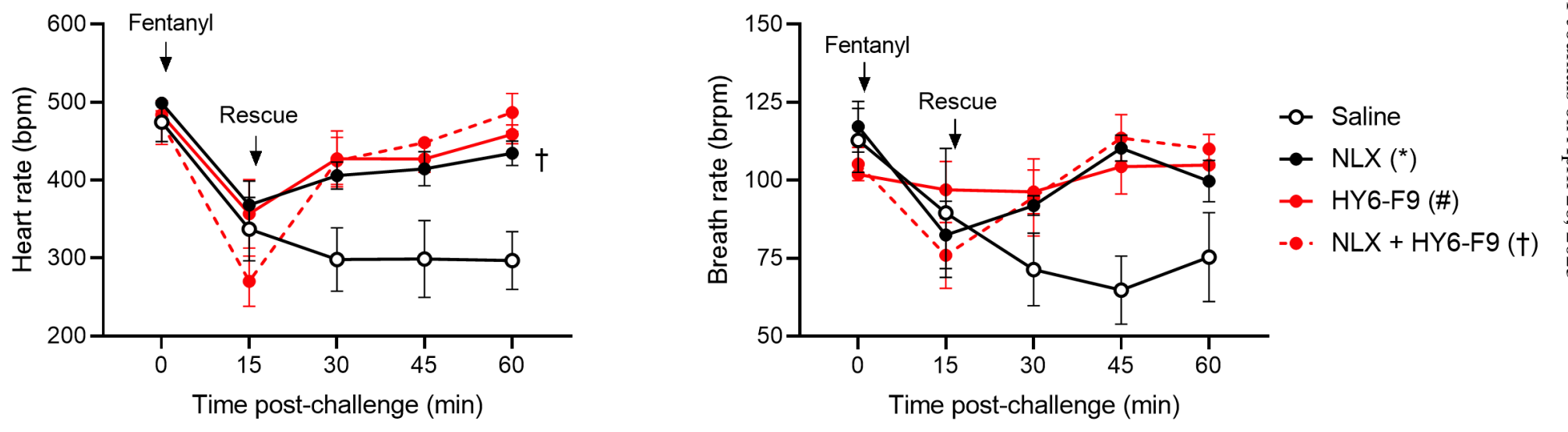
Figure 4

A
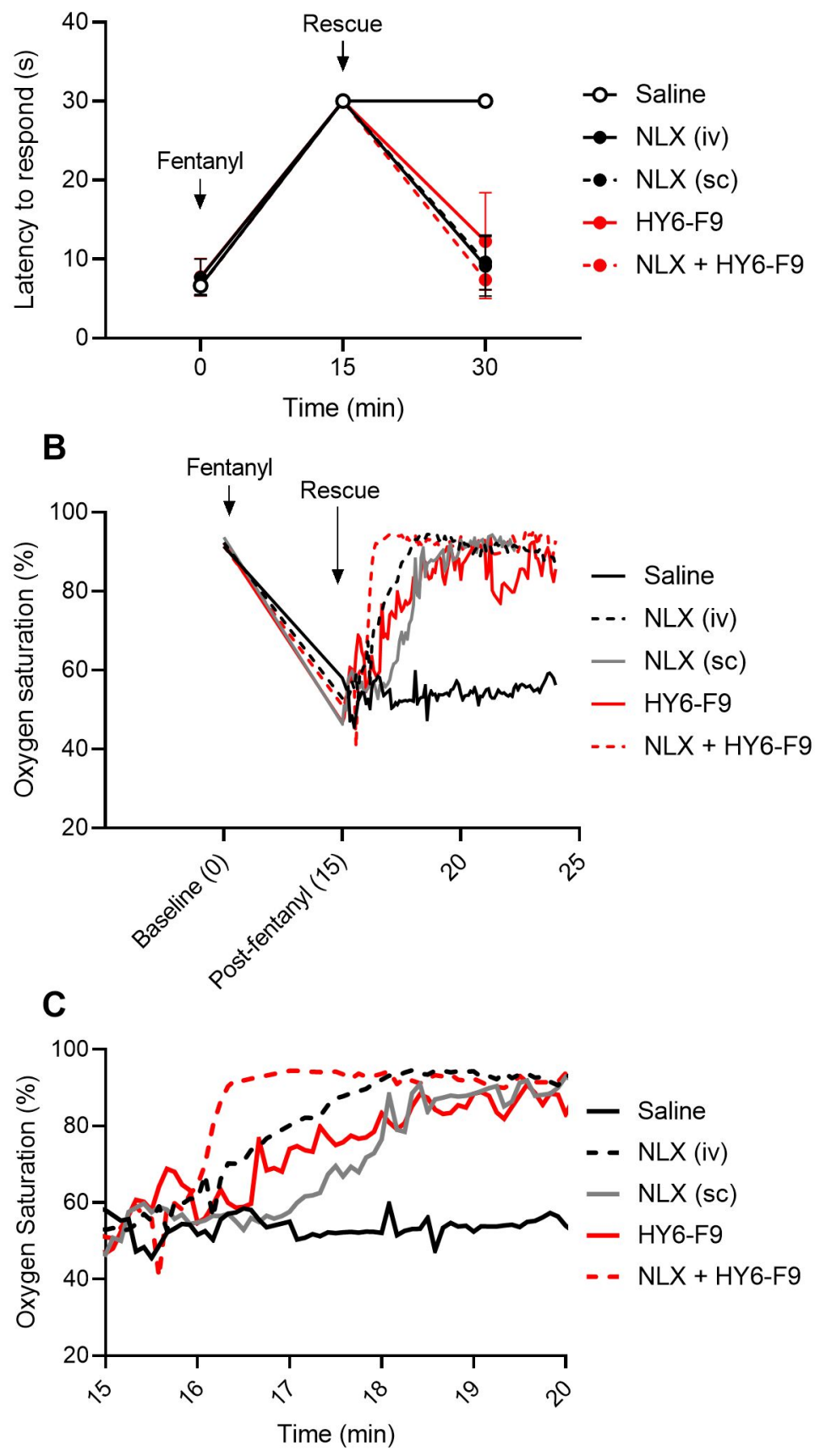


\section{Figure 5}

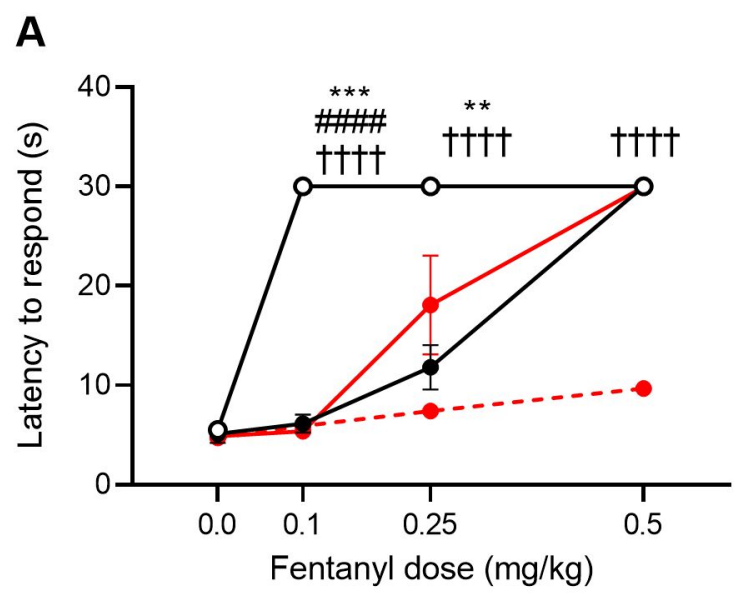

C

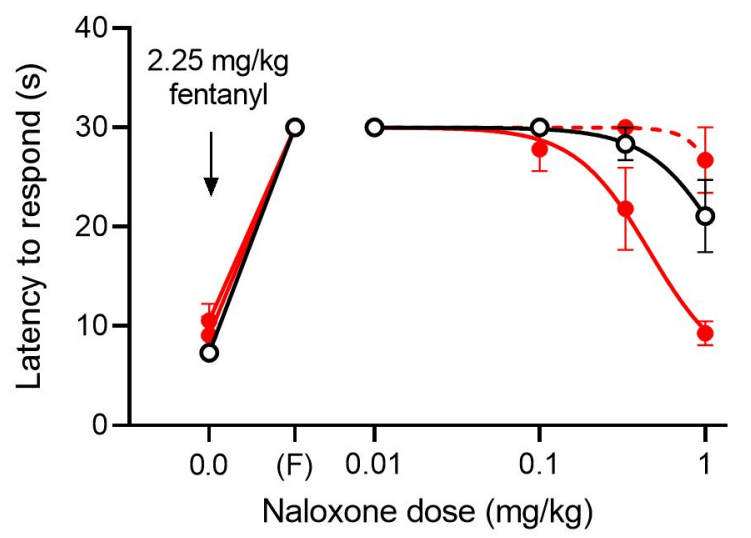

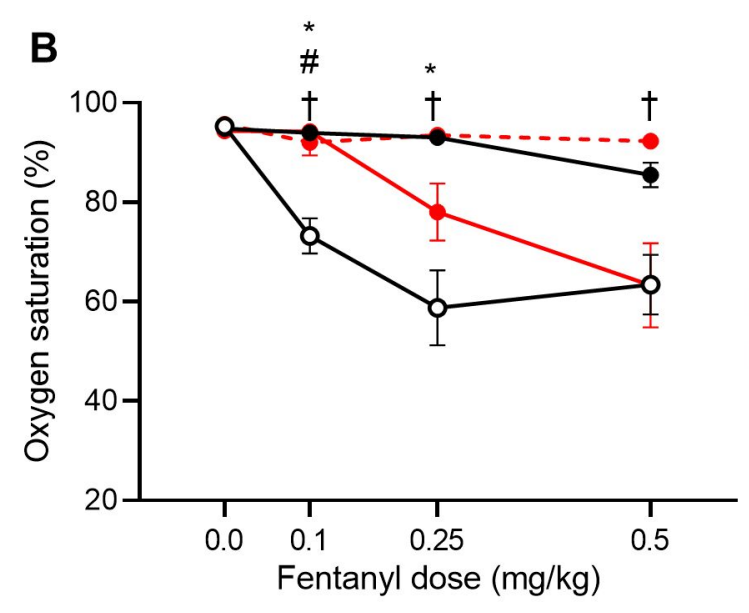

-o- Saline

$\rightarrow$ NLX $(*)$

$\rightarrow$ HY6-F9 (\#)

๑. NLX+HYG-F9 (†)

D

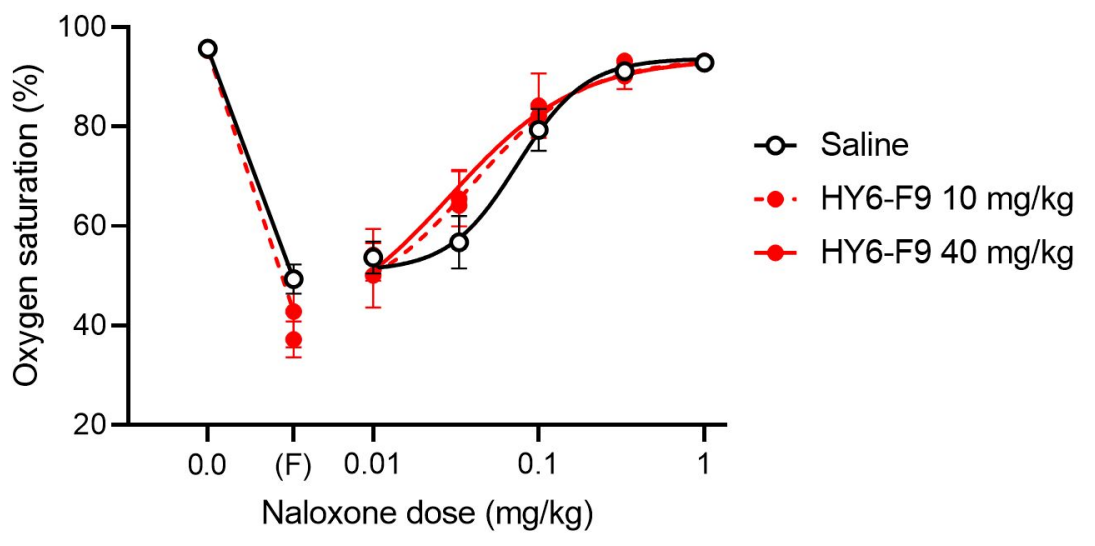




\section{Figure 6}
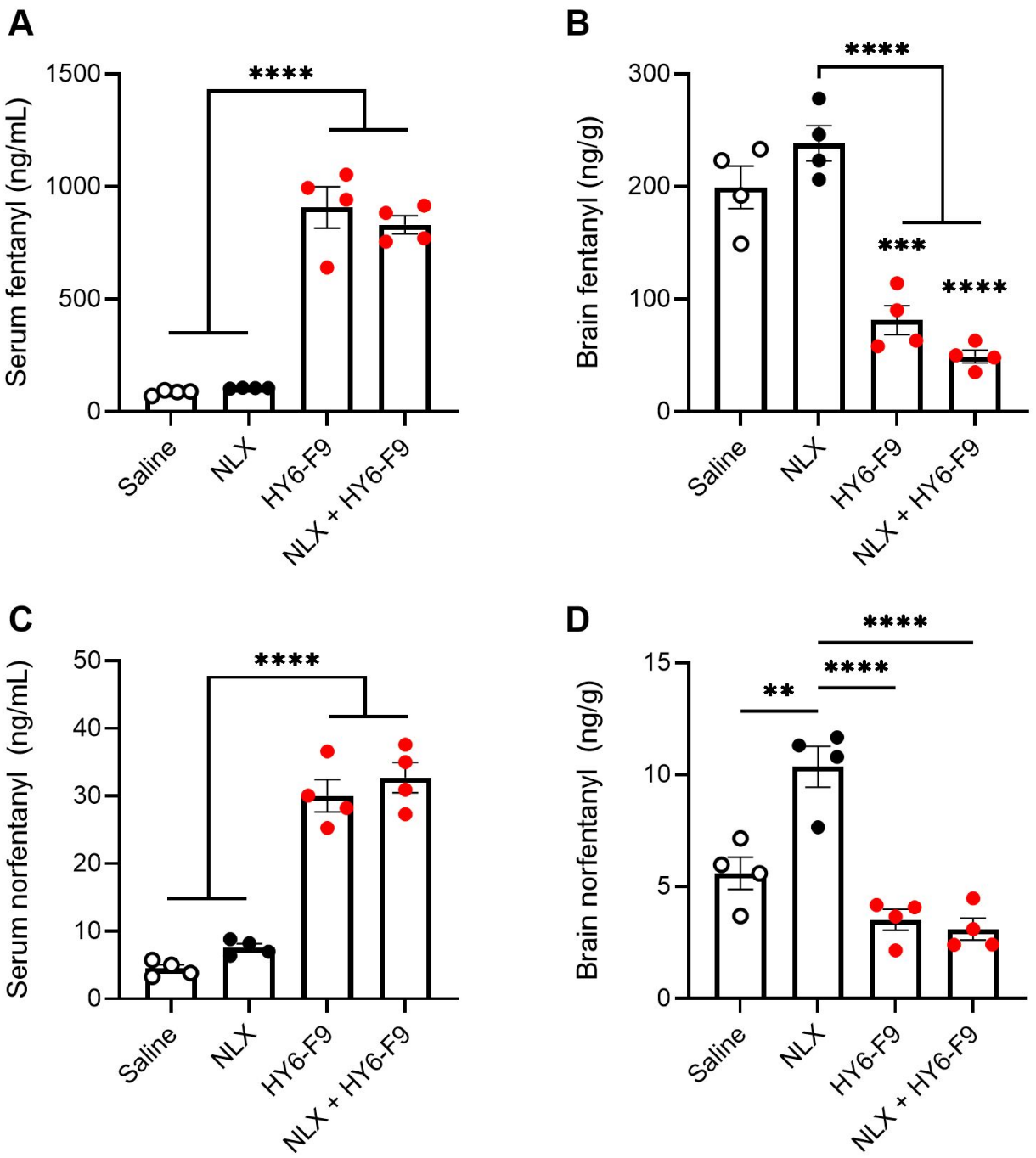